\title{
Betriebliche Effizienzgrößen für Ridepooling-Systeme
}

Prof. Dr. Christian Liebchen (TH Wildau), Prof. Dr. Martin Lehnert (TH Wildau), Dr. Christian Mehlert (KCW GmbH), Dr. Martin Schiefelbusch (NVBW GmbH)

\section{Inhalt}

Inhalt I

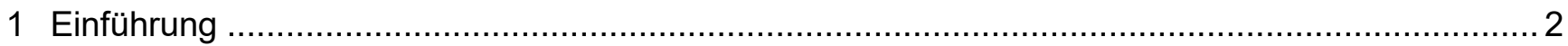

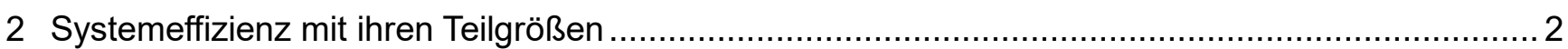

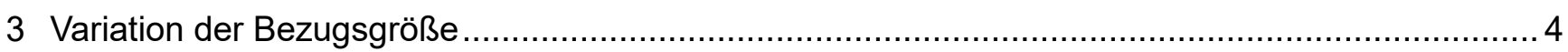

4 Systemeffizienz und Pooling-Index................................................................................. 5

5 Ausgewählte Vergleichswerte für andere Verkehrsmodi ..................................................... 7

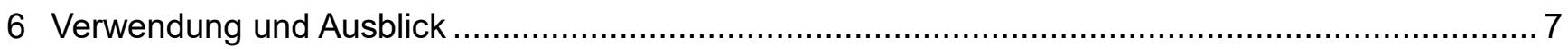

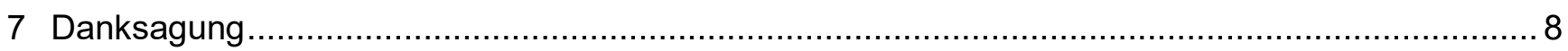

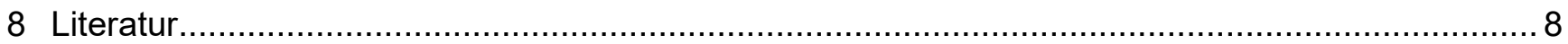

\section{PREPRINT}

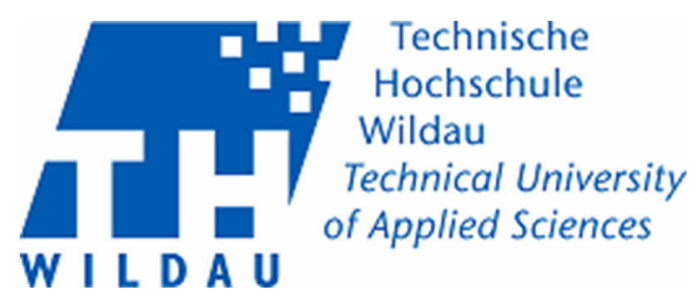

Vollversion angenommen zur Veröffentlichung im Tagungsband zum

"Wissenschaftsforum Mobilität 2020“

Universität Duisburg-Essen, Springer Verlag

Lizenz: CC-BY-NC-ND 


\section{$1 \quad$ Einführung}

Ridepooling stellt eine viel diskutierte Mobilitätsform dar. Ad-hoc und in der Regel von mobilen Endgeräten übermittelte Fahrtwünsche einzelner Fahrgäste werden bedarfsgesteuert, fahrplanfrei und vollflexibel zu möglichst effizienten, geteilten Fahrten gebündelt, welche im Regelfall mit Vans oder Kleinbussen durchgeführt werden. Sowohl aus Betreibersicht als auch insbesondere aus der Sicht von Genehmigungsbehörden stellt sich dabei die Frage nach der betrieblichen Effizienz von Ridepooling-Systemen, welche nicht zuletzt für die Bewertung der Konformität zu öffentlichen Verkehrsinteressen, speziell einer effizienten Nutzung öffentlicher Verkehrsinfrastruktur, relevant sein können. In diesem Zuge werden teilweise auch bereits Aspekte wie Umwegfaktor, Besetzungsgrad und Leerkilometeranteil diskutiert.

Im vorliegenden Beitrag werden diese Aspekte als ein Teilergebnis der Arbeit des FGSV-Arbeitskreises 1.6.1 „Verkehrliche Anforderungen an Ridepooling-Systeme" zu einem Kennzahlensystem zusammengefügt, die vollständigen Ausführungen finden sich dabei in Liebchen et al. (2020). Verschiedene Varianten der für dieses System vorgeschlagenen Top-Kennzahl "Systemeffizienz" haben dabei stets gemein, dass in innen der erzielte verkehrliche Nutzen durch den Gesamtaufwand zur Bereitstellung der hierfür benötigten Kapazitäten dividiert wird. Mit zunehmender Anwendung dieser strukturierten Vorgehensweise ließen sich Ridepooling-Systeme künftig sowohl gebiets- als auch anbieterübergreifend in ihrer betrieblichen Effizienz miteinander vergleichen. Entsprechend könnten die in dieser Arbeit vorgestellten Größen im Idealfall mit dazu beitragen, die von der Findungskommission zur Novellierung des Personenbeförderungsgesetzes (PBefG) zunächst allgemein formulierten Anforderungen („Vorgabe eines Besetzungsgrades“ bzw. einer „Poolingquote“, Berücksichtigung von Betriebsfahrten) konkret zu untersetzen (NaNa-Brief vom 09.06.2020).

In Abschnitt 2 stellen wir die Grundform der Kennzahl Systemeffizienz mit ihren Teilgrößen Umwegfaktor, Fahrzeugbesetzung und Leerkilometeranteil vor. Die in der Systemeffizienz gewählte Bezugsgröße für den Aufwand (Fahrzeugkilometer einschließlich Umwege und Leerkilometer) wird in Abschnitt 3 um verschiedene andere Aufwandsgrößen variiert. Abschnitt 4 betrachtet daneben im Gegensatz zur Systemeffizienz als Nutzengröße nicht mehr die für jeden Fahrgast einzeln gebuchte Streckenlänge, sondern berücksichtigt das Phänomen, dass Fahrgäste sich bereits im Zuge ihrer Buchung vorab selbst zu Gruppen bündeln. Dies führt auf die Größe PoolingIndex, welche eine günstigere Vergleichbarkeit speziell mit den Verkehrsmodi Taxi und Pkw-Verkehr bietet. Hiernach werden einzelne Referenzwerte, sowie ein Ausblick gegeben.

\section{Systemeffizienz mit ihren Teilgrößen}

Eine Möglichkeit zur Beschreibung von Effizienz ist die Division einer Nutzengröße durch eine Aufwandsgröße. Dieser Wert ist umso größer, je höher die Werte der Nutzengröße bzw. je geringer die Werte der Aufwandsgröße sind. Der Zweck eines Personenverkehrssystems besteht darin, Fahrtenwünsche mehrerer Personen von deren Start- zu deren Zielort zu realisieren. Die hierfür im Verkehrsmarkt etablierte Einheit sind entsprechend die Personenkilometer.

Bei den im öffentlichen Linienverkehr regelmäßig betrachteten betrieblichen Effizienzgrößen wird jedoch meist eine rein betriebliche und somit von Fahrgästen unabhängige Perspektive eingenommen. Zur Beschreibung betrieblicher Effizienz wird hier in aller Regel eine Nutzengröße durch eine Aufwandsgröße dividiert, so z. B. bei der 
Planungseffizienz (auch: Schichtproduktivität) als Quotient von Beförderungszeit durch Dienstplanzeit (Schnieder, 2015). So versteht sich eine Nutzengröße „Fahrplankilometer“ in dem im ÖPNV-Markt verbreiteten Besteller-Ersteller-Modell insbesondere im Sinne der Perspektive des Erstellers: Fahrplankilometer stellen auch unabhängig von der Auslastung mit Fahrgästen einen Nutzen dar, da für diese Bestellerentgelt erzielt wird.

Aufgrund der vorliegenden individuellen Buchungen lässt sich in Ridepooling-Systemen die Nutzengröße Personenkilometer sehr viel präziser fassen. Diese muss hier gleichwohl differenziert betrachtet werden. Denn um verschiedene Fahrtenwünsche bündeln („poolen“) zu können, werden teilweise Umwege gefahren. Diese generieren zwar zusätzliche tatsächlich gefahrene Personenkilometer, stellen jedoch keinen unmittelbaren Nutzen für den jeweiligen Fahrgast dar. Insofern beschreibt erst die Größe „Personenkilometer gebucht“ - also die bei direkter Fahrt erforderlichen Kilometer ohne etwaige bündelungsbedingt teilweise erforderliche Umwege - den tatsächlich von einem Ridepooling-System erbrachten Nutzen.

Insbesondere aus kommunaler Sicht besteht der Aufwand eines Ridepooling-Systems in der Intensität der Nutzung der Straßeninfrastruktur. Eine im Verkehrsmarkt übliche Einheit, um diese zu beschreiben, ist der Fahrzeugkilometer. Dabei ist es zunächst unerheblich, ob eine Fahrt mit oder ohne Fahrgäste stattfindet und ob es sich um den direkten Weg von Fahrgästen handelt, oder ob der Fahrtabschnitt für einen oder mehrere Fahrgäste einen Umweg darstellt (,all in“).

Das Verhältnis der Nutzengröße „Personenkilometer gebucht“ zu der Aufwandsgröße „Fahrzeugkilometer gesamt“ (alle Fahrzeugkilometer mit und ohne Fahrgäste inkl. aller Umwege und Leerfahrten insb. in Form von Ein-, Umsetz- und Aussetzfahrten) bildet damit die Grundform der Top-Kenngröße „Systemeffizienz“:

$$
\text { Systemeffizienz }=\frac{\text { Personenkilometer gebucht }}{\text { Fahrzeugkilometer gesamt }} \text {. }
$$

Im Weiteren werden als kleines Kennzahlensystem rechnerische Zusammenhänge zwischen der Systemeffizienz und ihren Teilgrößen Leerkilometeranteil, Umwegfaktor und Fahrzeugbesetzung vorgestellt (isoliert wurden diese auch von Werner und Karl, 2019, betrachtet). Diese dienen unmittelbar dazu, das Zustandekommen eines bestimmten Wertes für die Systemeffizienz besser zu verstehen.

Der Umwegfaktor, den die Fahrgäste in einem Ridepooling-System im Mittel erfahren haben, ergibt sich wie folgt:

$$
\text { Umwegfaktor }=\frac{\text { Personenkilometer gefahren }}{\text { Personenkilometer gebucht }} \text {. }
$$

Als zweite Größe zur Aufschlüsselung der Systemeffizienz eines Ridepooling-Systems wird sich die mittlere Fahrzeugbesetzung als passend erweisen, welche in Abgrenzung zu Leerfahrten nur für die mit mindestens einem Fahrgast besetzten Fahrtabschnitte ermittelt wird:

$$
\text { Fahrzeugbesetzung }=\frac{\text { Personenkilometer gefahren }}{\text { Fahrzeugkilometer besetzt }} \text {. }
$$

Schließlich ist noch der Leerkilometeranteil (Fahrzeugkilometer ohne Fahrgäste) in einem Ridepooling-System zu betrachten und lässt sich wie folgt ermitteln: 


$$
\text { Leerkilometeranteil }=\frac{\text { Fahrzeugkilometer leer }}{\text { Fahrzeugkilometer gesamt }} .
$$

Unter Berücksichtigung des folgenden Zusammenhangs zwischen leer und besetzt zurückgelegten Fahrzeugkilometern (Fzgkm)

$$
\text { Fzgkm gesamt }=\text { Fzgkm leer }+ \text { Fzgkm besetzt }
$$

ergibt sich die Kenngröße Systemeffizienz in ihrer Grundform anhand des folgenden rechnerischen Zusammenhanges zwischen den vorstehend vorgestellten Teilgrößen:

$$
\text { Systemeffizienz }=\frac{1}{\text { Umwegfaktor }} \cdot \text { Fahrzeugbesetzung } \cdot(1-\text { Leerkilometeranteil })
$$

bzw.

$$
\text { Systemeffizienz }=\frac{P k m \text { gebucht }}{\text { Pkm gefahren }} \cdot \frac{\text { Pkm gefahren }}{\text { Fzgkm besetzt }} \cdot \frac{\text { Fzgkm besetzt }}{\text { Fzgkm gesamt }} \text {. }
$$

Hieraus lassen sich unmittelbar die folgenden Abhängigkeiten ableiten:

- je höher der Umwegfaktor, desto geringer die Systemeffizienz,

- je höher die mittlere Fahrzeugbesetzung, desto höher die Systemeffizienz und

- je höher der Leerkilometeranteil, desto geringer die Systemeffizienz.

Diese Zusammenhänge machen deutlich, dass im Falle einer - sei es aus kommunaler oder aus Betreibersicht mangelhaften Systemeffizienz stets auch mindestens eine der drei Teilgrößen Leerkilometeranteil, Umwegfaktor und Besetzung nicht zufriedenstellende Werteniveaus besitzen muss. Nicht zuletzt sei explizit vermerkt, dass die Werte sämtlicher der hier betrachteten Basisgrößen aus den im Rahmen des Buchungs- und Flottenmanagements ohnehin verfügbaren Daten unmittelbar ermittelt werden können.

\section{Variation der Bezugsgröße}

Die im vorherigen Abschnitt eingeführte Grundform der Kenngröße Systemeffizienz kann dabei zu einzelnen Artefakten führen. So weist das in Tab. 1 beschriebene Ridepooling-System 1 zwar einen geringeren Umwegfaktor und einen geringeren Leerkilometeranteil als Ridepooling-System 2 auf; es führt aber aufgrund der niedrigeren Fahrzeugbesetzung zu einer geringeren Systemeffizienz.

\begin{tabular}{|c|r|r|}
\hline & Ridepooling System 1 & Ridepooling System 2 \\
\hline Systemeffizienz & 2 & 2,5 \\
\hline Teilgrößen & 1,2 & 1,5 \\
\hline Umwegfaktor & 3 & 5 \\
\hline Fahrzeugbesetzung & $20 \%$ & $25 \%$ \\
\hline Leerkilometeranteil &
\end{tabular}

Tab. 1: Gegenüberstellung der Systemeffizienz zweier Ridepooling-Systeme, sowie ihrer Teilgrößen

Solange System 2 eine entsprechend höhere Fahrzeugauslastung (Fahrzeugbesetzung in Bezug auf die im Fahrzeug verfügbare Platzkapazität) aufweist, mag ein solches Gesamtergebnis plausibel bleiben. Aber wenn in System 2 vergleichsweise große Fahrzeuge mit einer Kapazität von 12 Fahrgastplätzen eingesetzt werden, in System 1 hingegen nur Fahrzeuge mit Platz für bis zu 6 Fahrgäste, dann wäre selbst eine solche Begründung nicht zutreffend. Dieses Artefakt findet im Kern seine Ursache darin, dass in der Grundform der Kenngröße Systemeffizienz zunächst eine Normierung mit der Fahrzeuggröße ausgespart wurde. 
Für eine entsprechende Normierung sind dabei mehrere zur Größe Fahrzeugkilometer gesamt alternative Bezugsgrößen vorstellbar, welche bei Einsatz größerer Fahrzeuge auf jeweils höhere Werte im Nenner und somit auf kleinere Werte für die jeweilige Variante der Systemeffizienz führen:

- (Sitz-) Platzkilometer gesamt

- Pkw-Einheiten-Kilometer (vgl. FGSV 2015) gesamt

- Energiebedarf gesamt.

Für eine detaillierte Vorstellung dieser Varianten sei hier auf Liebchen et al. (2020) verwiesen.

\section{$4 \quad$ Systemeffizienz und Pooling-Index}

Eine zur Bewertung von Ridepooling-Systemen virulente Frage ist nicht zuletzt der Grad, zu dem verschiedene Buchungen (wobei eine Buchung z. B. die vier Mitglieder einer Familie umfassen kann) gebündelt („gepoolt“) produziert werden. Denn derzeit sind viele Ridepooling-Systeme in Deutschland unter der sog. „Experimentierklausel“ des §2 Abs. 7 PBefG genehmigt. Diese dient der „praktischen Erprobung neuer Verkehrsarten oder Verkehrsmittel". Würde in einem Ridepooling-System keine Bündelung von verschiedenen Buchungen geschehen, dann würde dieses System weitgehend in einem taxiähnlichen Modus operieren, so dass es fraglich wäre, ob die Schwelle der „praktischen Erprobung neuer Verkehrsarten“ in Abgrenzung zur etablierten Verkehrsart „Taxi“ überhaupt übersprungen würde.

In einer ersten einfachen Betrachtung kann eine sog. „Poolingrate“ bezogen auf die Zahl der gepoolten Fahrten berechnet werden, indem der prozentuale Anteil derjenigen Buchungen, deren Fahrgäste einen Teil ihres Weges zusammen mit Fahrgästen anderer Buchungen zurückgelegt haben, an der Gesamtzahl an Buchungen berechnet wird („fahrtbasierte Poolingrate“, Kirschbaum und Meyer, 2018). In Tab. 2 sind hierzu beispielhaft drei Buchungen dargestellt, die mit demselben Fahrzeug befördert werden und bei denen die Fahrgäste sämtlicher Buchungen jeweils eine (kurze) Strecke ihres Weges zusammen mit Fahrgästen anderer Buchungen zurücklegen. Wenn, wie im Beispiel, drei von drei Buchungen auf Teilstrecken gebündelt sind, dann ergibt sich entsprechend eine auf die Zahl der gepoolten Buchungen bezogene Poolingrate von 100\%.

\begin{tabular}{|r|r|r|r|}
\hline & Buchung 1 & Buchung 2 & Buchung 3 \\
\hline Strecke von A nach B & $8 \mathrm{~km}$ & --- & --- \\
\hline Strecke von B nach C & $1 \mathrm{~km}$ & $1 \mathrm{~km}$ & --- \\
\hline Strecke von C nach D & --- & $7 \mathrm{~km}$ & -- \\
\hline Strecke von D nach E & --- & $1 \mathrm{~km}$ & $1 \mathrm{~km}$ \\
\hline Strecke von E nach F & --- & --- & $8 \mathrm{~km}$ \\
\hline $\begin{array}{r}\text { Weglängen der Bu- } \\
\text { chungen }\end{array}$ & $9 \mathrm{~km}$ & $9 \mathrm{~km}$ & $9 \mathrm{~km}$ \\
\hline Gesamtfahrtstrecke & & $25 \mathrm{~km}$ \\
\hline
\end{tabular}

Tab. 2: Beispiel für drei Buchungen, die mit demselben Fahrzeug befördert werden und die - bezogen auf die Zahl der gepoolten Buchungen - auf eine Poolingrate von 100\% führen („Drei von drei Buchungen werden auf Teilstrecken gebündelt produziert.")

Diese Perspektive springt jedoch offensichtlich zu kurz, denn von den 25 Fahrzeugkilometern befanden sich nur auf 2 km (also 8\%) Fahrgäste aus verschiedenen Buchungen in dem Fahrzeug. Kirschbaum und Meyer (2018) 
regen hier entsprechend bereits an, beispielsweise eine Normierung anhand der Zeit vorzunehmen (,zeitbasierte Poolingrate").

Der Argumentation von Krijan (2020) und der Systematik der zuvor vorgestellten Systemeffizienz folgend nehmen wir hier eine Normierung anhand der Strecke vor und diskutieren Gemeinsamkeiten und Unterschiede mit der Systemeffizienz. Krijan (2020) definiert dabei den von inm so bezeichneten „Pooling-Index“ wie folgt:

$$
\text { PoolingIndex }=\frac{\text { Buchungskilometer ohne Umwege }}{\text { Fahrzeugkilometer gesamt }} \text {. }
$$

Der Pooling-Index würde angewendet auf das Beispiel aus Tab. 2 entsprechend nur den Wert $\frac{27}{25}=1,08$ betragen, wenn dort angenommen wird, dass alle Buchungen auf direktem Weg befördert wurden. In dem auf diese Weise definierten Pooling-Index kommt ungleich besser als in der fahrtbasierten Poolingrate zum Ausdruck, dass in diesem Beispiel in nur sehr geringem Umfang gepoolt wurde. Dies gilt insbesondere im Vergleich mit dem entsprechenden Wert von eins für Pkw-Verkehr, wenn bei letzterem zunächst vorübergehend vereinfachend Parkplatzsuchverkehr vernachlässigt wird.

Auch für diesen Pooling-Index ist im Grunde dieselbe Aufschlüsselung in Teilgrößen wie Umwegfaktor und Leerkilometer möglich. Hierzu ist zunächst die Beobachtung nützlich, dass sich bei der folgenden Rechnung derselbe Wert für den oben eingeführten Umwegfaktor ergibt:

$$
\text { Umwegfaktor }=\frac{\text { Buchungskilometer gefahren }}{\text { Buchungskilometer ohne Umwege }} \text {. }
$$

Für eine vollständige Aufschlüsselung in Teilfaktoren wird hier dann nur noch die folgende Teilgröße der mittleren Anzahl der Buchungen, die sich zeitgleich in einem besetzten Fahrzeug befinden („zeitgleiche Buchungen“), benötigt:

$$
\text { BuchungenZeitgleich }=\frac{\text { Buchungskilometer gefahren }}{\text { Fahrzeugkilometer besetzt }} .
$$

Der Pooling-Index schlüsselt sich damit in die folgenden Teilgrößen als Faktoren auf:

$$
\text { PoolingIndex }=\frac{1}{\text { Umwegfaktor }} \cdot \text { BuchungenZeitgleich } \cdot(1-\text { Leerkilometeranteil }) \text {. }
$$

Die frappierende Gemeinsamkeit mit der Grundform der Systemeffizienz zeigt sich auch in dem rechnerischen Zusammenhang zwischen dieser und dem Pooling-Index. Als Bindeglied ist lediglich die - mit Kilometern gewichtete - mittlere Anzahl von Personen je Buchung zu definieren:

$$
\text { PersonenJeBuchung }=\frac{\text { Personenkilometer gebucht }}{\text { Buchungskilometer ohne Umwege }} \text {. }
$$

Mit dieser ergibt sich der folgende rechnerische Zusammenhang zwischen Systemeffizienz und Pooling-Index:

$$
\text { Systemeffizienz }=\text { PersonenJeBuchung } \cdot \text { PoolingIndex. }
$$


Hieraus wird ersichtlich, dass die Systemeffizienz umso höher ist, je stärker sich die Fahrgäste bereits vorab selbst „poolen“, also in Buchungen zusammenfassen (Familie, Freunde). Der Pooling-Index erweist sich diesem Effekt gegenüber immun.

\section{$5 \quad$ Ausgewählte Vergleichswerte für andere Verkehrsmodi}

Für die Berechnungsvorschrift der Größen „Personenkilometer gebucht“ und „Buchungskilometer ohne Umwege“ stellt sich generell die Frage, ob diese entlang des bezüglich der Zeit oder entlang des bezüglich der räumlichen Entfernung kürzesten Weges ermittelt werden sollten. Die Wahl der räumlichen Entfernung würde dabei riskieren, regelmäßig verkehrlich generell nicht sinnvolle Routen als Bezugsgröße heranzuziehen, beispielsweise wenn diese mitten auf ihrem Weg kleine reine Erschließungsstraßen nutzen. Die Wahl des zeitlich schnellsten Weges als Grundlage für die Berechnung der beiden Größen wird dabei unmittelbar vom jeweiligen Buchungszeitpunkt abhängen. Dies mag zwar vernünftig erscheinen, kann jedoch dazu führen, dass im Falle einer sich ändernden Verkehrslage nun ein anderer Weg zeitlich kürzer wird als der zu Beginn berechnete kürzeste Weg. Dabei kann dann der neue Weg sogar eine kürzere Strecke aufweisen und somit Umwegfaktoren mit Werten strikt kleiner als eins generieren (siehe Liebchen et al., 2020).

Für einzelne der zuvor eingeführten Teilgrößen lassen sich für andere Verkehrsmodi klare Vergleichswerte identifizieren:

- Pkw-Verkehr - Leerkilometeranteil: 0,0

- Pkw-Verkehr - Umwegfaktor: minimal über 1,0 (Aufschlag innerstädtisch insbesondere für Parkplatzsuchverkehr)

- Pkw-Verkehr - zeitgleiche Buchungen: geringfügig unter 1,0

- Pkw-Verkehr - Fahrzeugbesetzung: ca. 1,36 (MiD, 2018)

- Taxiverkehr - Umwegfaktor: 1,0

- Taxiverkehr - zeitgleiche Buchungen: 1,0

Beim Vergleich mit Linienverkehr fehlt in Erhebungen regelmäßig ein Bezug zu den von mehreren Personen gemeinsam zurückgelegten Wegen („Buchungen“). Zudem erweist es sich hier als nicht-trivial, geeignete Entsprechungen für das Phänomen von Umwegen zu entwickeln. Von daher muss an dieser Stelle auf die Angabe entsprechender Vergleichswerte verzichtet werden.

Durch die ausschließliche Fokussierung auf Buchungen unabhängig von der Personenanzahl in jeder Buchung bietet der Pooling-Index hier klarere Vergleichsmöglichkeiten mit den beiden Verkehrsmodi Taxiverkehr und PkwVerkehr:

- Ridepooling vs. Taxiverkehr: Um wie viel überwiegt der Ridepooling-Vorteil zeitgleicher Buchungen seinen aus Umwegen resultierenden Nachteil?

- Ridepooling vs. Pkw-Verkehr: Um wie viel überwiegt der Ridepooling-Vorteil zeitgleicher Buchungen seine aus Umwegen und Leerkilometern resultierenden Nachteile?

\section{$6 \quad$ Verwendung und Ausblick}

Die oben dargestellte Berechnung der Systemeffizienz entstand insbesondere vor dem Hintergrund der Bewertung aktueller Ridepooling-Systeme im Vergleich zum Pkw- und Taxiverkehr in Großstädten. Diese in aller Regel nicht öffentlich bestellten Ridepooling-Systeme tragen meist nicht gezielt zur Daseinsvorsorge im Sinne des §8 Abs. 3 
PBefG bei. Um einen Beitrag zur Verkehrswende durch Verkehrsentlastung im motorisierten Autoverkehr leisten zu können, wären für ein Ridepooling-System sicher größere Werte für die Systemeffizienz notwendig, als sie in den vergleichbaren Größen für Taxi- aber auch für Individualverkehr erreicht werden. Zudem gilt überdies, dass je größer die Systemeffizienz eines Ridepooling-Systems ist, desto größer ist dessen Potenzial verkehrsentlastend wirken zu können.

Ridepooling-Systeme können aber auch gezielt im Rahmen der Daseinsvorsorge eingesetzt werden, nicht zuletzt wenn eine nur geringe Nachfrage den Einsatz von Linienbussen mit attraktiven Fahrtenabständen haushaltsseitig nur schwer rechtfertigen würde. Dies ist beispielsweise in den dünner besiedelten ländlichen Räumen der Fall, aber ggf. auch in Randlagen größerer Städte. Hier besteht meist ein geringeres Poolingpotenzial, sowie längere Leerfahrten. Diese führen zwangsläufig zu niedrigeren Werten für die Systemeffizienz, so dass an diese hier weniger hohe Ansprüche zu richten wären.

Im Zusammenhang mit der derzeit diskutierten Reformierung des Personenbeförderungsgesetzes wird in Hinblick auf die Genehmigung von Ridepooling auch die Einführung einer Kenngröße „Poolingquote“ diskutiert. Mit ihr soll gemessen werden, ob die lokalen Ziele zur Verkehrsvermeidung tatsächlich erreicht werden und Ridepooling zu weniger (Straßen-) Verkehr beiträgt. Über die exakte Definition der Poolingquote, wie auch die Berücksichtigung u.a. von „Leerfahrten und Suchverkehren“, wird aktuell offenbar noch gestritten (NaNa-Brief vom 09.06.2020). Es würde die Autoren freuen, wenn die hier vorgeschlagene Systemeffizienz mit ihren Teilgrößen Eingang in die Diskussion zur Definition adäquater betrieblicher Effizienzgrößen finden würde.

Auch bei der längerfristigen Perspektive des möglichen eigenwirtschaftlichen Einsatzes sehr vieler fahrerloser Fahrzeuge wären aus kommunaler Sicht stets solche Anbieter zu bevorzugen, welche die für das Segment Ridepooling dann bestehende Verkehrsnachfrage mit möglichst großen Werten in der Größe Systemeffizienz befriedigen.

\section{$7 \quad$ Danksagung}

Die Autoren bedanken sich herzlich bei den Mitgliedern des Arbeitskreises 1.6.1 „Verkehrliche Anforderungen an Ridepooling-Systeme" der Forschungsgesellschaft für Straßen- und Verkehrswesen (FGSV) und bei den Studierenden des Moduls "Mobility Concepts“ an der Technischen Hochschule Wildau im Wintersemester 2019/2020 für ergiebige Diskussionen.

\section{$8 \quad$ Literatur}

Forschungsgesellschaft für Straßen- und Verkehrswesen (2015): „Handbuch für die Bemessung von Straßenverkehrsanlagen“, ISBN 978-3-86446-103-3, FGSV-Verlag Köln

Kirschbaum, Tom; Meyer, Sabrina (2018): „Revolution im Ridesharing - Pooling könnte viele Pkw ersetzen“, In: bahn manager, ISSN 2367-1998, 01/2018, S. 56-61.

Krijan, Ivica (2020): „Saturday Night Pooling - Pooling am Samstag den 16.11.2019“, https://dieklage.de/pooling-index-7.html, abgerufen am 19.05.2020

Liebchen, Christiian; Lehnert, Martin; Mehlert, Christian; Schiefelbusch, Martin: „Betriebliche Effizienzgrößen für Ridepooling-Systeme“, Tagungsband zum Wissenschaftsforum Mobilität 2020, Universität Duisburg-Essen, Springer-Verlag, akzeptiert zur Veröffentlichung

MiD (2018), Nobis, Claudia; Kuhnimhof, Tobias: Mobilität in Deutschland - MiD Ergebnisbericht. Studie von infas, DLR, IVT und infas 360 im Auftrag des Bundesministers für Verkehr und digitale Infrastruktur. Bonn, Berlin. www.mobilitaet-in-deutschland.de

NaNa-Brief (2020): „Streit um Poolingquote“, NaNa-Brief vom 09.06.2020, DVV Media Group GmbH, Hamburg

Schnieder, Lars (2015): „Betriebsplanung im öffentlichen Personennahverkehr“, ISBN 978-3-662-46455-7, Springer-Verlag 